\title{
Impact of fiber versus steel ropes on the lifetime of crane winches
}

\author{
Mohamed Yousri Georg Jacobs Stephan Neumann
}

RWTH Aachen University, Institute for Machine Elements and System Engineering, Schinkelstr. 10, DE-52062 Aachen, Germany. E-mail: \{yousri.mohamed,georg.jacobs,stephan.neumann\} @imse.rwth-aachen.de

\begin{abstract}
Offshore crane winches are equipment installed on sea vessels and designed for accurate lifting and lowering of payloads at subsea levels in all conditions. In recent times, attempts have been made to replace conventional heavy steel ropes used in crane winches with high-strength synthetic fibre ropes for their various advantages, particularly for having comparable strength with steel at a much lower weight. Such an advantage makes it possible, in principle, to downsize a crane winch for the same lifting capacity leading to considerable savings or alternatively, to increase the service life of an existing crane winch and make the most out of its lifting capacity. However, it is still not known to what extent can replacing steel ropes in crane winches with fibre ropes increase their service life and in this paper, an attempt is made to answer this question.

In previous research, there have been attempts to calculate the service life of crane winches, however without taking the dynamics of the ropes they utilise and their material into account. In this paper, the global loads that a typical offshore crane winch drivetrain is subject to and its resulting service life are analysed and compared in both cases of using steel and fibre ropes, by deriving the global loads on the drivetrain directly from the tension that is calculated in the ropes for a typical operational cycle using a rope dynamics simulation model. The conclusion of this work, subject to further analysis, is that fibre ropes lead to a significant reduction in static torque (global load) on the winch drivetrains, due to their light weight and a reduction in the dynamic torque due to their lower stiffness compared to steel ropes. Consequentially, crane winches would benefit from a higher service life which is of critical importance due to their remoteness and difficulty of access for maintenance.
\end{abstract}

Keywords: Lifetime, Reliability, Offshore, Winch, Fibre, Ropes

\section{Introduction}

Offshore crane winches are equipment installed on sea vessels and designed for accurate lifting and lowering of payloads at subsea levels in all conditions, including extremely cold temperatures down to $-40^{\circ} \mathrm{C}$ with working depths of up to $4,000 \mathrm{~m}$ and a safe working load capacity of up to 600 tonnes (single line) (MacGregor, 2019) and are usually equipped with AHC (Active Heave Compensation) capabilities that control the vertical movement of the payload such that its oscillation due to waves is kept to an absolute minimum or at zero and thus the payload, at a given depth, is motionless in relation to the seabed. Offshore winch cranes with AHC can be supplied with electric or hydraulic drives to provide the required torque to compensate for wave motion. A visual representation of AHC can be seen in Figure 1.

In recent times, attempts have been made to replace 


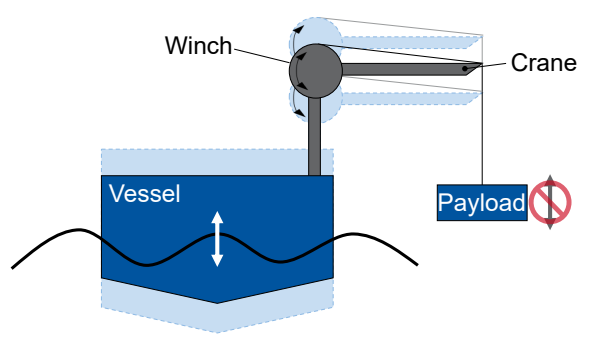

Figure 1: Visual representation of AHC (Wll et al., 2018)

conventional heavy steel ropes in crane winches with high-strength fibre ropes made of high-strength synthetic fibres such as aramid fibres (HMPA), aramid/carbon fibre mixtures or high-modulus polyethylene fibres (HMPE) (Baldinger et al., 2018). Fibre ropes are believed to be able to unlock the full lifting potential of a crane winch, because they are virtually weightless when submerged in water and therefore no matter how much rope is paid out, this does not add any significant extra load to the crane winch and so larger payloads can be lifted at large depths than what was possible with steel ropes. In Table 1, it can be seen from the parameters of rope A and rope $\mathrm{C}$ that fibre ropes can have a similar or higher breaking strength than steel ropes with a much lower specific mass, and therefore fibre ropes can match steel ropes in their mechanical properties as well. The superior flexibility of fibre ropes allows for smaller bending radii and thus smaller rope sheaves, leading to further weight reduction in other components of the crane winch (Baldinger et al., 2018). Another advantage of fibre ropes is that they do not need to be lubricated in order to avoid high friction like when using steel ropes, and are thus more environmentally friendly.

In addition to the advantages mentioned above, fibre ropes have the potential of reducing dynamic loads on the crane winch drivetrain due to their generally lower stiffness. The reduction in static load caused by a reduction in the total submerged weight of the rope and payload when using fibre ropes as well as the reduction of dynamic load due to lower stiffness, makes it possible in principle to downsize the drivetrain or increase the service life of existing drivetrains, thus potentially leading to considerable cost savings in the design and operation thereof, given that the higher cost of fibre ropes does not take up much of the saved expense.

It is still unknown, however, the extent to which using fibre ropes in crane winches can lead to an increase in the service life of crane winch drivetrains.
Therefore, it would be of great relevance to know what influence does changing to fibre ropes have on the loads experienced by the crane winch drivetrain, and consequently on the lifetime of it and its components?

To answer these questions, the lifetime of a typical offshore crane winch with 150 tons lifting capacity is analysed in this paper utilising different ropes described in Table 1, of which rope $\mathrm{A}$ is made of steel and ropes $\mathrm{B}$ and $\mathrm{C}$ are made of UHMWPE. The payload which is carried by the rope is assumed to be stable at 3,000 $\mathrm{m}$ depth and only oscillating due to the effect of waves, with the oscillations damped due to $64 \%$ AHC. In order to obtain the global load that the crane winch gearbox is subject to and which is the main factor in determining its lifetime, the tension response in the ropes has to be calculated, which differs from one rope to the other depending on its material and size.

The lifetime calculation is based on the methodology outlined in (Neumann et al., 2016) and utilises a code suited specifically to calculate the lifetime of components in a gearbox typically used in crane winches with a 150 tons lifting capacity, being able to calculate the lifetime of oscillating bearings in the gearbox, as described in (Wll et al., 2018). Since taking the effect of rope dynamics and different rope materials into account in the lifetime calculation of a crane winch is something that has not been done yet, this paper addresses this area in order to provide more clarity about the effects of replacing steel ropes with fibre ropes.

\section{Methods}

In the subsections below, the rope dimensions as well as the methods used to calculate rope dynamics (oscillation and tension), rope dynamic stiffness and crane winch lifetime are described respectively.

\subsection{Rope dimensions}

The properties of ropes A, B and C used in calculations carried out in this paper are shown in Table 1. Of these three variants, ropes $\mathrm{A}$ and $\mathrm{B}$ are typical steel and fibre ropes used in deepwater lowering operations, both having similar lengths and diameters. Rope $\mathrm{C}$ is the same material as Rope B, with a bigger diameter and used for comparative purposes. UHMWPE is an abbreviation for Ultra-High Molecular Weight Polyethylene material, and is a subset of thermoplastic polyethylene with extremely long molecular chains and a high molec- 
Yousri et al., "Impact of fiber versus steel ropes on the lifetime of crane winches"

Table 1: Dimensions and material properties of ropes A, B and C

\begin{tabular}{lccc}
\hline Parameter & \multicolumn{3}{c}{ Rope } \\
\cline { 2 - 4 } & Rope A & Rope B & Rope C \\
\hline Commercial name & CASAR Powerplast & Dyneema SK75 & Dyneema SK75 \\
Material & Steel & UHMWPE & UHMWPE \\
$L:$ Length $[\mathrm{m}]$ & 3,000 & 3,000 & 3,000 \\
$D:$ Diameter $[\mathrm{m}]$ & 0.072 & 0.072 & 0.096 \\
$E$ : Youngs modulus $[\mathrm{MPa}]$ & $2 \mathrm{E} 5$ & $1.13 \mathrm{E} 5$ & $1.13 \mathrm{E} 5$ \\
$m_{p}:$ Specific mass $[\mathrm{kg} / \mathrm{m}]$ & 25.49 & 2.54 & 4.51 \\
$\rho_{r}$ : Density $\left[\mathrm{kg} / \mathrm{m}^{3}\right]$ & 7,850 & 970 & 970 \\
$M B L_{p}:$ Breaking strength $[\mathrm{MPa}]$ & $5,047.7$ & $3,072.4$ & $5,191.0$ \\
\hline
\end{tabular}

ular mass (Kurtz, 2004), which results in a very tough, very strong material.

\subsection{Rope dynamics calculation}

Due to the motion of waves, the vessel onto which the crane winch is installed moves in 6 DOF as shown in Figure 2, which causes the crane tip (see Figure 1) to move in $\mathrm{x}, \mathrm{y}$ and $\mathrm{z}$ directions. The vertical component of the crane tip motion induces an oscillatory motion of the rope and the payload installed at the end of the rope. The vertical motion of the crane tip can be derived from the complex motion of the vessel.

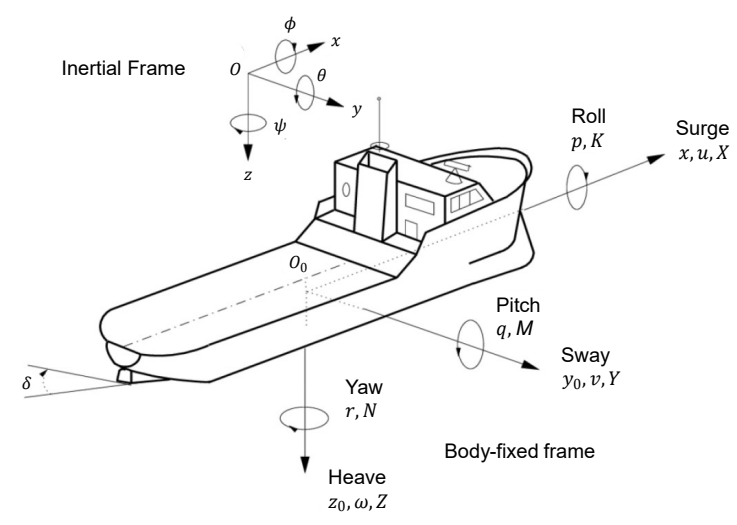

Figure 2: 6 DOF motion of a vessel due to waves (SNAME, 1950)

The wave sample used in the calculations is representative of deep-water waves in the Beaufort Sea. The wave sample has been obtained using JONSWAP spectrum developed by Hasselmann et al. (1973), after analysing data collected during the Joint North Sea Wave Observation Project (JONSWAP). The sample is made up of 18 wave propagations, spanning 1 hour, and from which the crane tip oscillation and rope tension will be calculated for 1 hour as well.
From the rope tension sample, the torque on the winch drum is calculated using expression (14) and is meant to act as synthetic representatives of the actual global load, from which the lifetime model will extrapolate in order to calculate the failure probability of the crane winch for a simulation time of $10^{6}$ hours. The reason behind using synthetic load samples and extrapolating them is that data sets are not available for a complete life cycle. An approach to modelling synthetic load spectra is presented by Wll et al. (2017b)

In order to calculate the oscillatory motion as well as dynamic tension at any given point in the steel or fibre ropes, the recommended practice DNVGL-RPN103 (Modelling and analysis of marine operations) (DVNGL, 2017) was used.

The vertical motion $\eta(z)$ of a straight vertical rope along with the lifted object installed at its end, at any vertical position $\mathrm{z}$ of the rope caused by a forced oscillation of the crane tip with frequency $\omega$ and amplitude $\eta_{a}$ can be calculated with equation (1). The complex wave number $k$ is calculated using equation (3a).

$$
\frac{\eta(z)}{\eta_{a}}=\left|\frac{k E A \cos [k(z+L)+h(\omega) \sin [k(z+L)]]}{k E A \cos (k L)+h(\omega) \sin (k L)}\right|
$$

Due to this oscillatory motion of the rope and payload, a dynamic tension $F_{d}(z)$ is induced in the rope and its amplitude can be calculated at any position $\mathrm{z}$ of the rope with equation (2).

$$
\frac{F_{d}(z)}{\eta_{a} k_{E}}=\left|\frac{-(k L)^{2} k_{E} \sin [k(z+L)]+F^{\prime}}{(k L) k_{E} \cos (k L)+h(\omega) \sin (k L)}\right|
$$


Whereby,

$$
\begin{gathered}
k=\sqrt{\frac{m_{p}}{k_{E} L} \sqrt{\omega^{2}-i \omega\left(\frac{\sigma}{m_{p}}\right)}} \\
h(\omega)=-\omega^{2} M^{\prime}+i \omega \Sigma \\
F^{\prime}=(k L) h(\omega) \cos [k(z+L)]
\end{gathered}
$$

Other parameters in the previous equations are defined in the nomenclature in the beginning of the paper.

\subsection{Dynamic stiffness of fibre rope}

One of the major differences between synthetic fibre ropes and steel ropes is that the tension-elongation relation in fibre ropes is non-linear and time-dependent Flory et al. (2004), while the tension-elongation relation of steel ropes can be considered linear. Especially under cyclic loading (tension) induced by the combined effects of wind, wave and current as seen in Figure 3, the stiffness of fibre ropes changes from its initial quasi-static stiffness. The dynamic stiffness can be thought of as the stiffness of a fibre rope after applying fast cyclic load to it. Therefore, since the tension experienced by the rope is of a fast cyclic nature, the dynamic stiffness of ropes $\mathrm{B}$ and $\mathrm{C}$ have to be calculated.

Even though the usage of fibre ropes in offshore crane winches is recent, fibre ropes have been used in mooring applications for quite a while now, pioneered by Petrobras in the 1990s with a number of guidelines and standards being published for fibre rope deepwater moorings. Since deepwater fibre mooring lines are also subject to cyclic tension due to waves, and especially during storms, there is a lot of literature attempting to describe the tension-elongation behaviour of fibre ropes under such conditions such as (Leech et al., 2003), (Casey and Banfield, 2002) and others that are mentioned further in this text. The methods by which this behaviour can be described include a springdashpot model as described in (Falkenberg et al., 2019), deriving empirical expressions by performing tests on sample ropes as well as more complex computational models which account for internal abrasion and creep to accurately predict the service-life of the ropes (Flory et al., 2005).

Since the main focus in this paper is on the lifetime evaluation of crane winches with fibre ropes, empirical expressions found in literature are the best compromise between accuracy and computational expense. Several empirical expressions have been derived from tests carried out by different researchers throughout the years in order to describe the dynamic stiffness behaviour of a rope in response to cyclic loading. However, all of these expressions are only fit to the specific types of rope tested. From these tests, the main loading conditions which were found to affect the evolution of dynamic stiffness of a fibre rope are the mean load, load/strain amplitude and number of load cycles.

Francois and Davies (2000) and Wibner et al. (2003) have proposed expression (4) for calculating the dynamic stiffness of polyester ropes.

$$
K_{r}=\alpha+\beta L_{m}
$$

Here, $L_{m}$ is the mean load and $\alpha$ and $\beta$ are material property coefficients. Francois and Davies (2000) have derived the upper and lower bounds for coefficients $\alpha=15-18.5$ and $\beta=0.25-0.33$. Wibner et al. (2003) have derived the upper and lower bounds for coefficients $\alpha=17-23$ and $\beta=0.2-0.25 . \quad K_{r}$ here is a non-dimensional dynamic stiffness.

For ropes made of aramid, Davies et al. (2002) have proposed the following expression (5).

$$
K_{r}=45.0+0.58 L_{m}
$$

While for high-modulus polyethylene (HMPE) ropes, Davies et al. (2002) also proposed an empirical expression to calculate dynamic stiffness as follows in (6).

$$
K_{r}=59.0+0.54 L_{m}
$$

All these expressions have in common that they do ignore the effect of strain amplitude and number of loading cycles on the dynamic stiffness of fibre ropes. However, there exists a paper by Liu et al. (2014) in which an empirical expression was derived taking into account the three factors based on tests made on polyester, aramid and HMPE ropes using the mooring analysis method. This expression is written as follows in $(7)$.

$$
K_{r}=\alpha+\beta L_{m}-\gamma \epsilon_{a}-\delta e^{-k N}
$$

Here, $\epsilon_{a}$ is the strain amplitude, $\mathrm{N}$ is the number of loading cycles the rope is subject to, $\alpha, \beta, \gamma, \delta$ and $\mathrm{K}$ are coefficients pertaining to the construction of the specific fibre rope in study and the material it is made of, and coefficients $\delta$ and $\mathrm{K}$ are particularly 
Yousri et al., "Impact of fiber versus steel ropes on the lifetime of crane winches"

Table 2: Rope material coefficients for ropes tested in (Liu et al., 2014)

\begin{tabular}{cccccc}
\hline Material & \multicolumn{5}{c}{ Coefficient } \\
\cline { 2 - 5 } & $\alpha$ & $\beta$ & $\gamma$ & $\delta$ & $K$ \\
\hline Polyester & 7.16 & 0.41 & 6.30 & 1.78 & 0.010 \\
Aramid & 64.07 & 0.37 & 62.35 & 8.87 & 0.020 \\
HMPE & 53.56 & 0.62 & 50.70 & 8.24 & 0.013 \\
\hline
\end{tabular}

used to reflect the effect of long-term cyclic loading on the dynamic stiffness, something which is not taken into account in previous expressions derived by other researchers. Table 2 shows the values of these coefficients. Since this expression is one of the latest and most comprehensive that can be found in the literature, and it does not ignore the proven time-dependency of fibre rope stiffness under cyclic loading, it is used in this paper to calculate the dynamic stiffness of fibre ropes.

Since ropes B and C studied in this paper can be classified under high-modulus polyethylene (HMPE) ropes, the coefficients pertaining to HMPE ropes will be used. In this case, the expression used to calculate the dynamic stiffness is written as follows.

$$
K_{r}=53.56+0.62 L_{m}-50.70 \epsilon_{a}-8.24 e^{-k N}
$$

However, the dynamic stiffness value resulted from this expression is non-dimensional and in order to make it dimensional, it is treated as follows.

$$
K_{d t}=K_{r}\left(\frac{M B L_{t}}{L}\right)
$$

$M B L_{t}$ is the minimum breaking force of the test rope used in (Liu et al., 2014). Another thing to note is that the dynamic stiffness resulted from expression (9) is still only suitable for the dimensions of the HMPE rope tested in (Liu et al., 2014), and therefore a similarity criterion must be applied to derive the dynamic stiffness expression for our ropes B and C analysed in this paper.

A similarity criterion has been proposed by Fernandes and Rossi (2005) which is based on the fact that for two ropes of the same material but different size, the specific modulus $E / \rho$ is the same since the specific modulus is purely a material property.

$$
\lambda_{\frac{E}{\rho}}=\frac{\left(\frac{E}{\rho}\right)_{p}}{\left(\frac{E}{\rho}\right)_{t}}=\frac{\lambda_{M B L}}{\lambda_{m}}=1
$$

The subscript $p$ stands for prototype rope (ie. rope $\mathrm{B}$ or $\mathrm{C}$ used in this paper) and subscript $t$ stands for test rope tested in (Liu et al., 2014). $\lambda_{M B L}$ is the scale factor of the minimum breaking load and $\lambda_{m}$ is the scale factor of the specific masses of the ropes. From expression (10) it follows that,

$$
\frac{\lambda_{M B L}}{\lambda_{m}}=\frac{\frac{M B L_{p}}{M B L_{t}}}{\frac{m_{p}}{m_{t}}}=1
$$

and

$$
m_{p}=m_{t}\left(\frac{M B L_{p}}{M B L_{t}}\right)
$$

Here, $m_{p}$ is the specific mass of rope $\mathrm{B}$, calculated using the similarity criterion from the specific mass $m_{t}$ of the test rope used in (Liu et al., 2014) as well as the minimum breaking forces of both the prototype rope and the test rope. Likewise, another derivation from expression (12) leads to,

$$
K_{d p}=K_{d t}\left(\frac{m_{p}}{m_{t}}\right)=K_{r}\left(\frac{M B L_{t}}{L}\right)\left(\frac{m_{p}}{m_{t}}\right)
$$

With expression (13), the dimensional dynamic stiffness of rope $\mathrm{B}$ or $\mathrm{C}$ with a unit $[\mathrm{N} / \mathrm{m}]$ can be calculated directly from the non-dimensional dynamic stiffness $K_{r}$ of the test rope used in (Liu et al., 2014). The iteration procedure to calculate the dynamic stiffness of a fibre rope using expressions (7) and (13) is outlined in (Huang et al., 2015) and is followed in this paper. Figure 3 shows how the dynamic stiffness of ropes $\mathrm{A}, \mathrm{B}$ and $\mathrm{C}$ changes with increasing number of cycles. The fibre ropes $\mathrm{B}$ and $\mathrm{C}$ are assumed to already be worked and bedded-in before the calculation, having exceptional creep resistance such that long-term creep is negligible. Since the lifetime model calculates the failure probability of the crane winch on the long term for a very large number of cycles, the stiffness for each rope will be taken at its respective steady-state value. 


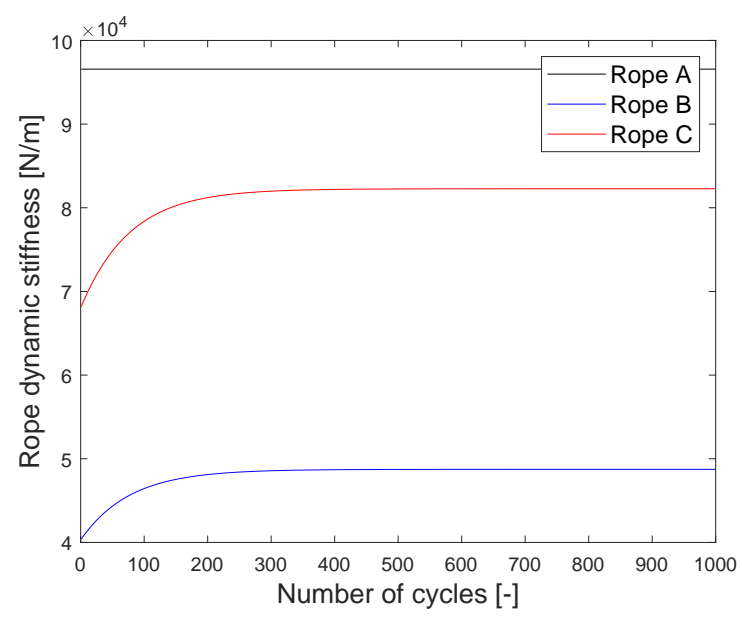

Figure 3: Influence of loading cycles on dynamic stiffness $K_{d} p$ for all ropes

\subsection{Connecting rope dynamics with lifetime calculation}

The lifetime calculation is outlined as follows, using the workflow outlined in (Neumann et al., 2016):

1) The torque which acts on the winch drum is taken as the global load. This torque is calculated by simply multiplying the tension $(F)$ at the end of the rope connected to the crane tip by the radius $(r)$ of the winch drum as shown in expression (14).

$$
T=F * r
$$

2) The resulting torque is divided equally onto the gearboxes on each side of the winch drum and the component stresses of each gearbox component are computed from the global load. A simplified schematic of the winch drum and gearbox is shown in Figure 4 .

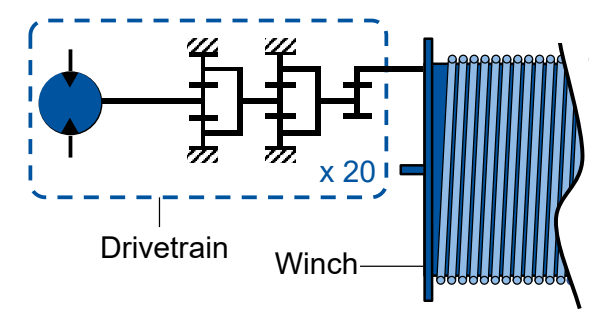

Figure 4: Simplified schematic of winch drum and drivetrain analysed (Wll et al., 2017b)

3) The component stresses of each component in the winch gearbox are classified using a counting method suited for oscillating bearings, developed by Wll et al. (2018). The load spectra produced by these counting methods are then compared to the bearable loads of the bearings in the gearbox calculated using (DIN ISO 281) for bearing fatigue, and the bearable loads of the gears in the gearbox calculated using (DIN 3390) for tooth strength and pitting resistance. The total damage induced in the components for a given load cycle is then calculated using the Palmgren-Miner damage accumulation theory (Miner, 1945). Figure 5 shows this theory in a schematic.

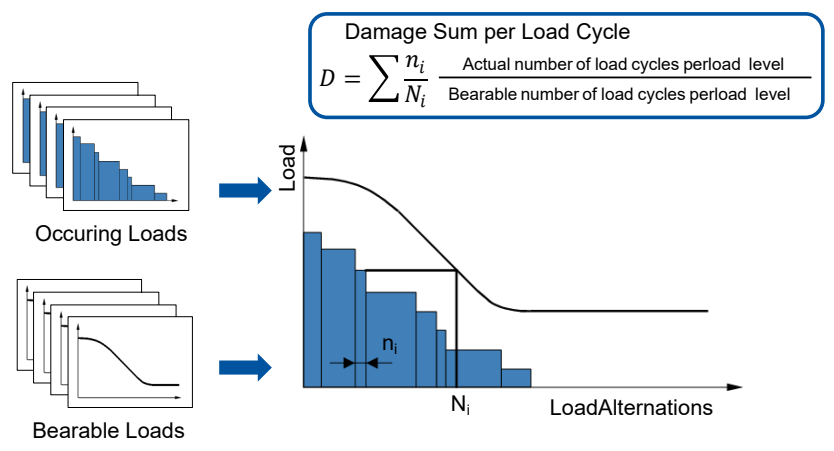

Figure 5: Schematic describing Palmgren-Miner damage accumulation theory (Miner, 1945)

4) The lifetime of each component, as well as the whole system is then calculated from the damage sum and is displayed as failure probability graphs as seen in Figure 6 .

\section{Results}

In this section, the lifetime of the winch drivetrain when using different ropes is shown, after which the torque (global load) that the winch drivetrain is subject to is shown when utilising different ropes. A sensitivity analysis is then made to identify the rope parameters that affect the lifetime of the winch drivetrain the most. Here, the payload mass is assumed to be constant at 75 tons and the rope mass is variable with the type of rope used.

\subsection{Lifetime results}

The failure probability graphs for the crane winch drivetrain when using different ropes are shown in Figure 6 . The increase in lifetime resulted from changing from rope $\mathrm{A}$ to rope $\mathrm{B}$, for example, is $1,435 \%$ due to an obvious total mass reduction when using fibre rope. This is discussed more in the Discussion section. 


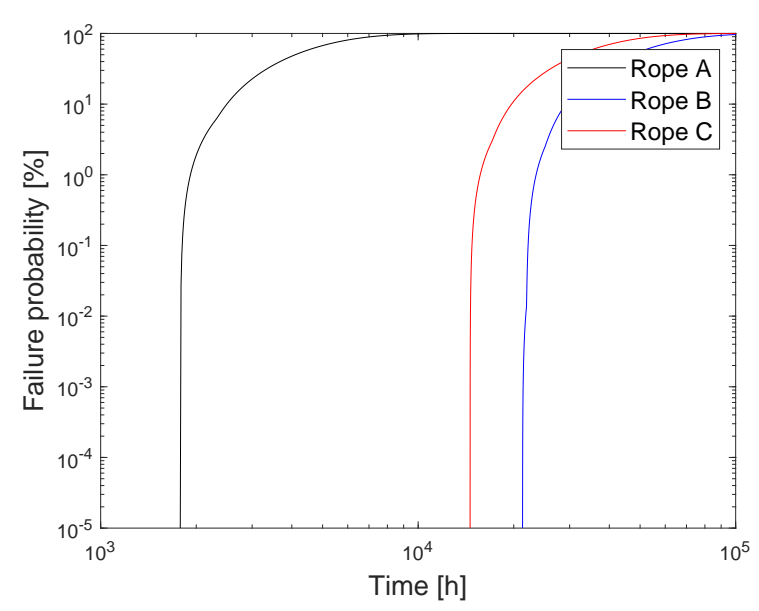

Figure 6: Failure probability of winch drivetrain utilising ropes $\mathrm{A}, \mathrm{B}$ and $\mathrm{C}$

\subsection{Global loads}

The torque (global load) that the winch drivetrains experience, and which is calculated from the rope tension at the crane tip using expression (14) is shown in Figure 7 and Figure 8, where in Figure 7, 18 cycles of the torque using ropes $\mathrm{A}, \mathrm{B}$ and $\mathrm{C}$ respectively can be seen from top to bottom and in Figure 8, 1 cycle of the same can be seen.

\subsection{Sensitivity analysis}

To study the effect of the most important parameters on the lifetime of the crane winch, a sensitivity analysis was made. As a general rule, the static tension in the rope as well as the amplitude and frequency of the dynamic tension are the main factors influencing lifetime, since the tension in the rope is directly proportional to the global load (torque) which the winch drivetrains are subject to as seen in expression (14). As seen in equation (2), the dynamic tension (and therefore dynamic torque) is, among other factors, dependent on the rope stiffness $k_{E}$. As for the static tension at the crane tip, its equation (15) is seen below and can be basically explained as the sum of the submerged weight of the rope and the payload attached at its end.

$$
F_{s}=\left(M g-\rho_{w} g V\right)+\left(m_{p} g-\rho_{w} g A\right) * L
$$

As seen in the equation, the static tension (and therefore static torque) depends on the payload mass $\mathrm{M}$ and the rope specific mass $m_{p}$, but since the goal is to study the effect of changing rope parameters on lifetime, we would only consider the effect of change of $m_{p}$ on the lifetime. Therefore, sensitivity analysis is carried out for:

1) $k_{E}$ vs. $T_{d}$ amplitude

2) $k_{E}$ vs. normalized lifetime

3) $m_{p}$ vs. $T_{s}$

4) $m_{p}$ vs. normalized lifetime

Where $T_{d}$ is the dynamic torque, $T_{s}$ is the static torque and the normalized lifetime is taken as the lifetime at a given point as a percentage of the lifetime at the first data point. The results of this analysis are shown in Figures 9 and 10. They are then discussed in the Discussion section.

\section{Discussion}

From Figure 6, it can be seen that the system benefits from a significantly higher lifetime when replacing steel rope in a crane winch with fibre rope due to a large reduction in total mass of the rope and payload. Speaking more specifically about the loads experienced by the drivetrain, when looking at Figure 7, it can be seen that the static torque as well as the amplitude of dynamic torque when using rope B are considerably lower than those of rope A, leading to a lower global load that the winch gearbox components are subject to. This can be pinpointed as the main reason why the lifetime of the winch increases when using rope $\mathrm{B}$ (or rope $\mathrm{C}$ as well). From Figure 10, it can be understood that the lower static torque for rope $\mathrm{B}$ is resulted from a much lower specific mass $(2.54 \mathrm{~kg} / \mathrm{m})$ than that of rope A $(25.49 \mathrm{~kg} / \mathrm{m})$. Likewise, from Figure 9, it can be understood that because rope $\mathrm{B}$ has a lower stiffness than rope $\mathrm{A}$, the amplitude of dynamic tension is lower and therefore this is another reason that contributes to a higher lifetime when using rope B.

The relation between rope stiffness $k_{E}$ and amplitude of $F_{d}$ is generally linearly proportional and with lifetime is generally linearly inversely proportional, but because the dynamic tension $F_{d}$ in equation (2) is dependent on other parameters such as the complex wave number $k$, which in itself is dependent on $k_{E}$ and $m_{p}$, the lifetime can deviate slightly from the linear behaviour, mainly because the frequency and amplitude of $F_{d}$ are affected by a change in the complex wave number $k$. As for the relation between rope specific mass $m_{p}$ and lifetime, it resembles closely a decaying exponent, with small deviations due to the same effect. The equation for $k$ is shown in (3a).

There is some degree of uncertainty however, from the results produced in this paper. Firstly, the results need to be validated using experimental results, which 


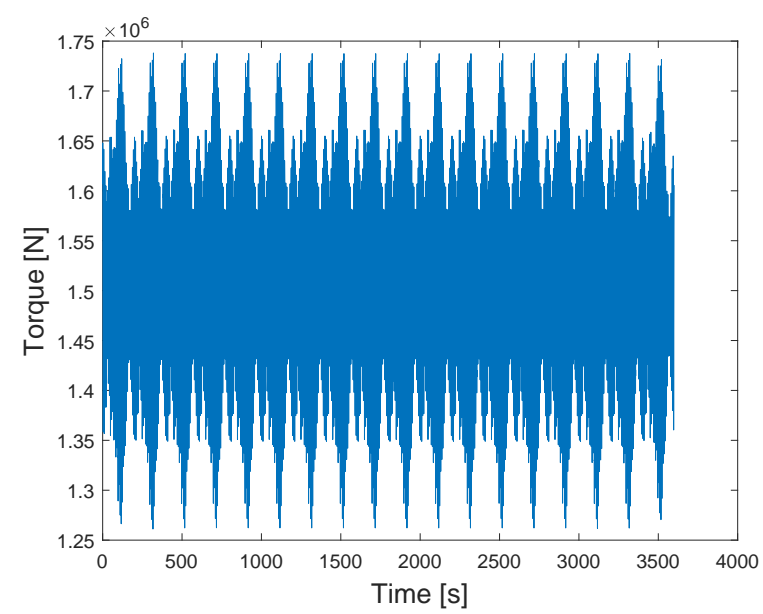

(a)

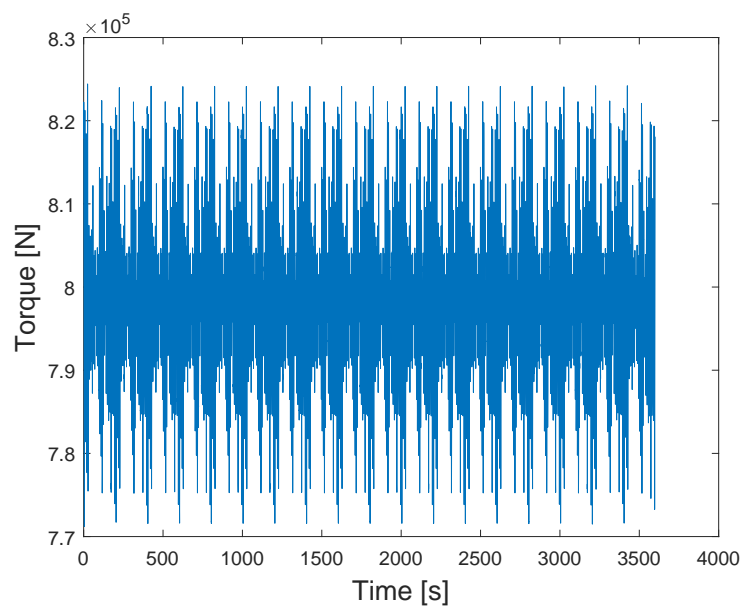

(b)

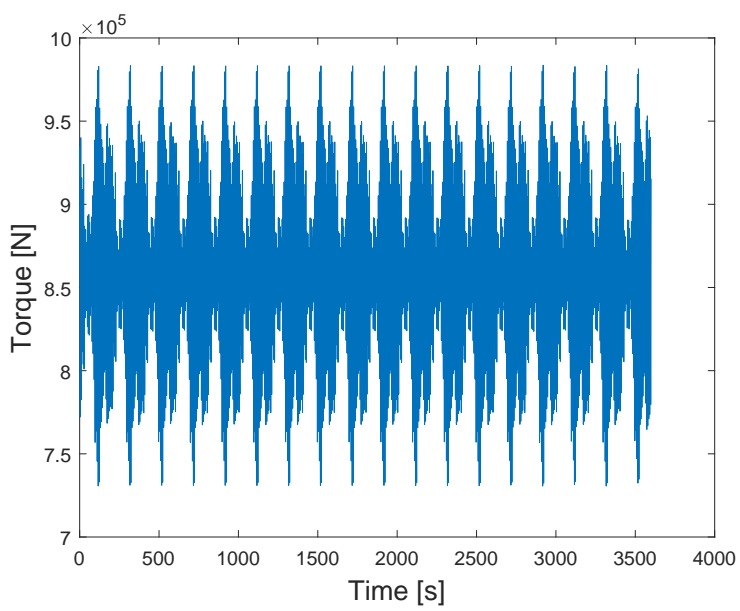

(c)

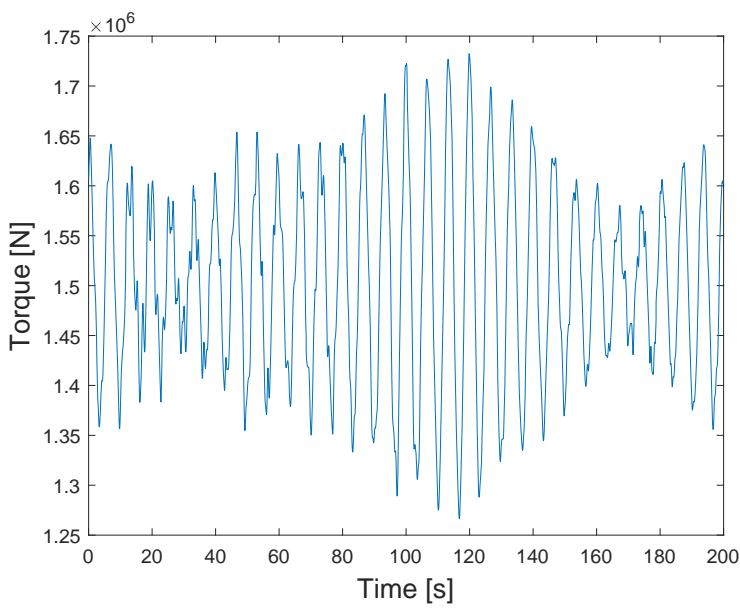

(a)

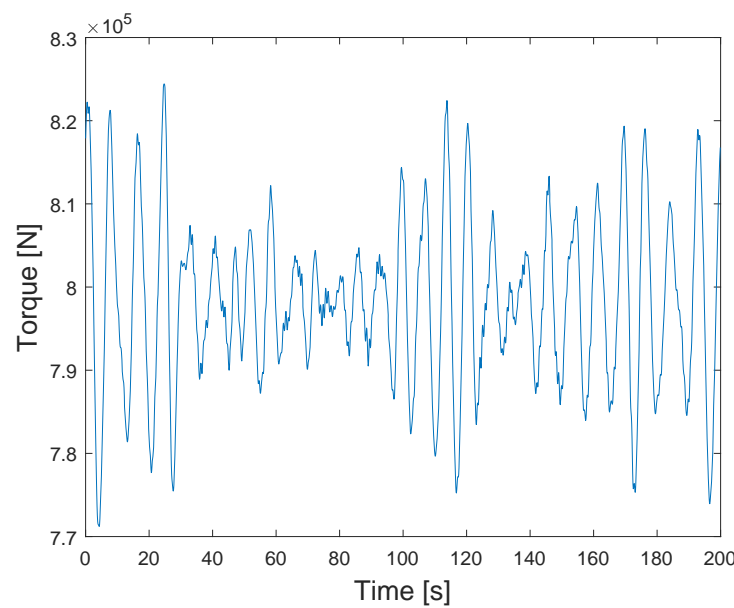

(b)

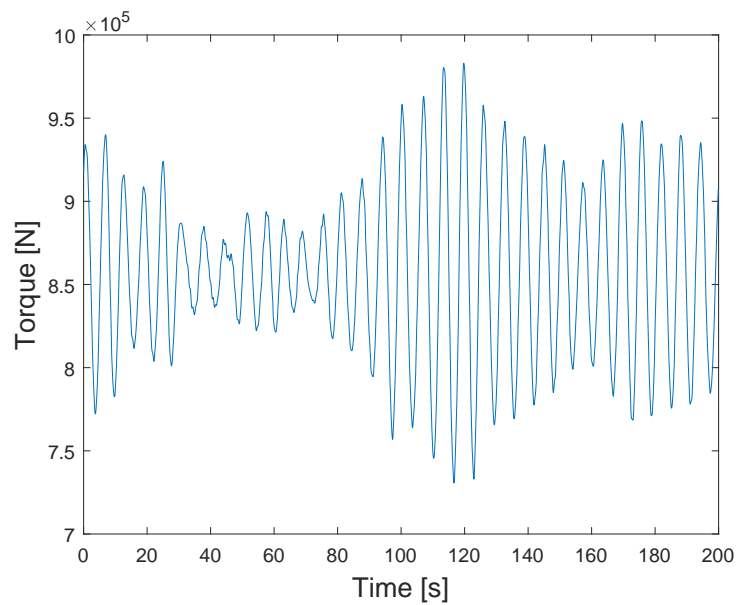

(c)

Figure 7: 18 cycles of torque on winch drivetrain us- Figure 8: 1 cycle of torque on winch drivetrain using ing Rope $\mathrm{A}, \mathrm{B}$ and $\mathrm{C}$ from top to bottom respectively Rope A, B and C from top to bottom respectively 


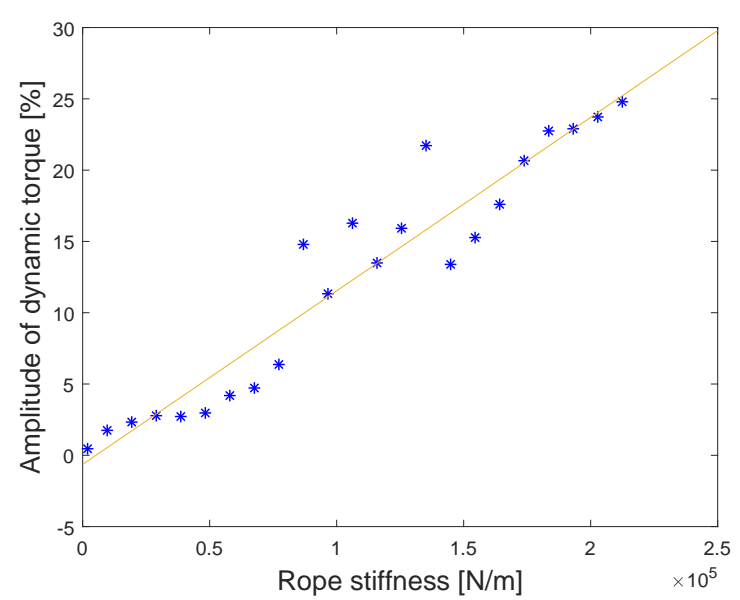

(a)

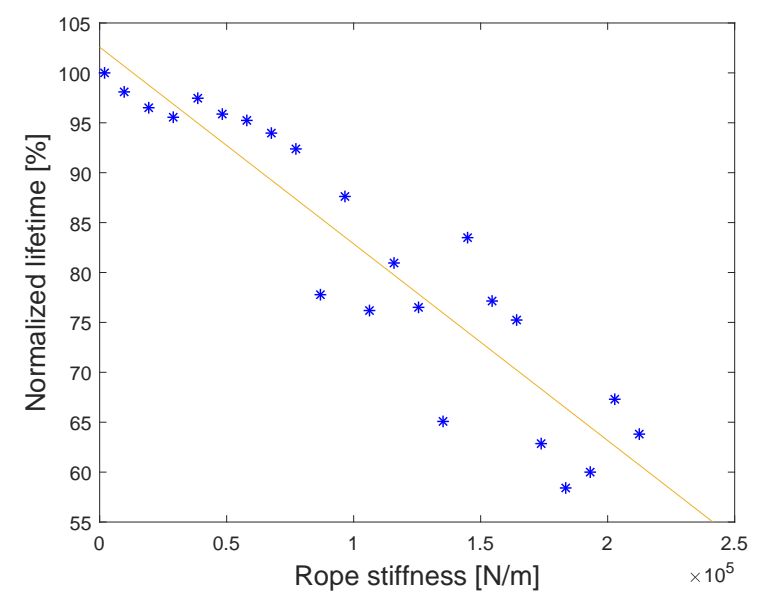

(b)

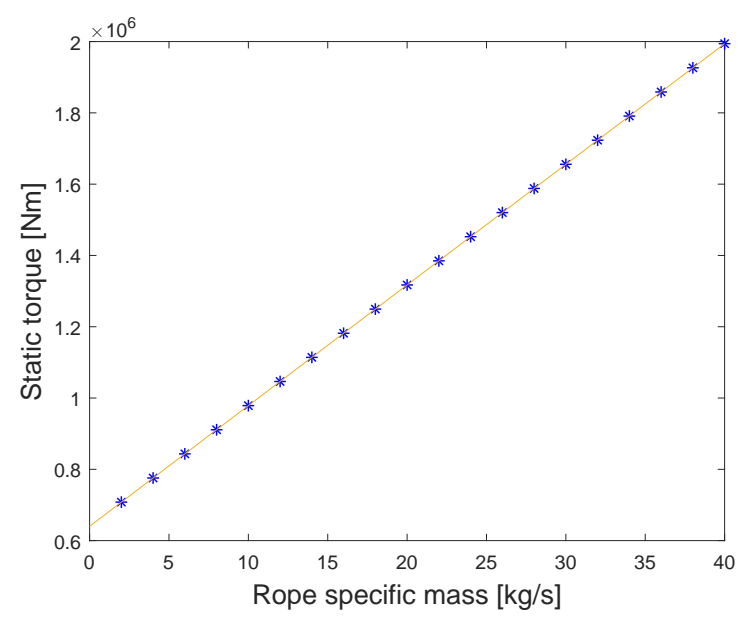

(a)

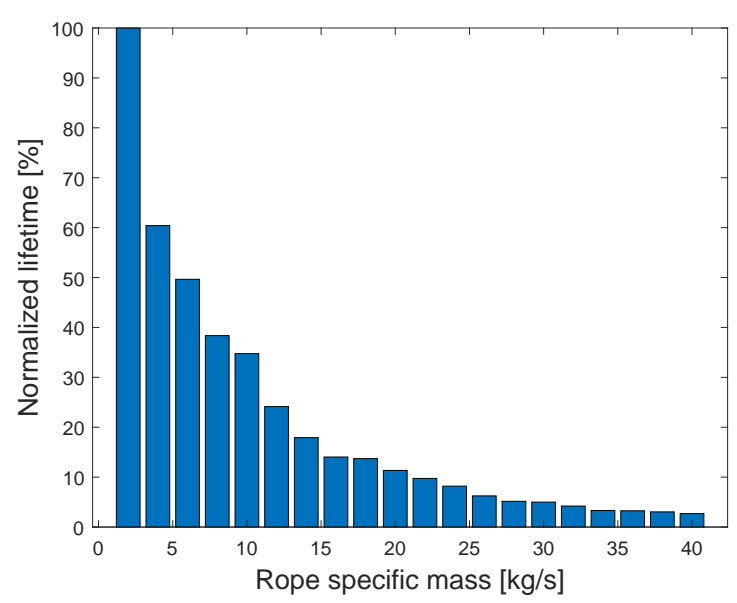

(b)

Figure 9: Effect of rope stiffness on amplitude of dy- Figure 10: Effect of rope specific mass on static torque namic torque (top) and normalized lifetime (bottom) (top) and normalized lifetime (bottom) 
is planned to be done in the near future. Secondly, the empirical expression (8) used to calculate the dynamic stiffness of fibre ropes under cyclic loading can only be used up to a certain level of accuracy for the specific type of rope material, construction and experimental conditions used in the tests carried out by Liu et al. (2014). Finally, further sensitivity analysis has to be made for other relevant rope parameters (for example, damping coefficient) in order to study their effect, the simulations have to also be run at different subsea depths and weather conditions and most importantly, it has to be checked if the enhanced AHC rotational speed requirement due to the fibre rope being more elastic than steel (and thus oscillating with larger amplitudes) will affect the large gain in lifetime seen in the results of this paper by introducing larger dynamic torque on the winch drivetrains. Other methods of connecting the rope dynamics with global load calculation shall be explored as well.

\section{Conclusion}

In this paper, the lifetime of a typical offshore crane winch with a 150 tons lifting capacity is compared using steel and fibre ropes in order to quantify the extent to which replacing steel ropes with fibre ropes in crane winches can lead to an increase in the service life of crane winch drivetrains. In a previous work by Wll et al. (2017a) on the reliability of offshore crane winches, the dynamic torque on the winch drivetrains is calculated from the acceleration and deceleration of the winch drum needed to coil and uncoil the rope to compensate for the payload oscillation due to waves, where the payload oscillation is assumed to be exactly equal to the wave motion. Up till here, the effect of rope material and dimensions on the torque was not considered. This current paper can be considered a continuation to this work, where the torque on the winch drivetrain is calculated directly from the tension in the rope, which is calculated using a rope dynamics model that takes the rope parameters into account so that the effect of different ropes on the lifetime of the drivetrain can be quantified.

From the results of this paper, an initial conclusion can be reached that fibre ropes lead to a significant reduction in static torque, due to their light weight and a reduction in the dynamic torque due to their lower stiffness, subject to further dynamic response analysis. Therefore, the global load on the winch drivetrains is considerably lower, leading to a consequential increase in the service life of the crane winch which is of critical importance due to their remoteness and difficulty of access for maintenance. This offers promising prospects of cost savings for beneficiaries due to longer availability of the equipment, as well as the ability to lift heavier payloads with the same crane winch they have. It also offers cost saving possibilities for manufacturers in the design phase of the crane winch, where they can downsize the drivetrain for the same lifting capacity.

\section{Acknowledgement}

The research presented in this paper has received funding from the Norwegian Research Council, SFI Offshore Mechatronics, project number 237896.

\section{References}

Baldinger, P., Traxl, R., Kaiser, G., Kirth, R., Ernst, B., and Rhrnssl, E. High-strength fiber ropes for lifting devices such as cranes. 2018. US Patent Application WO2017068054.

Casey, N. and Banfield, S. Full-scale fiber deepwater mooring ropes, advancing the knowledge of spliced systems. In Offshore Technology Conference Proceedings. Houston, TX, USA, 2002. Doi: https: //doi.org/10.4043/14246-MS.

Davies, P., Francois, M., Grosjaen, F., Baron, P., Salomon, K., and Trassoudaine, D. Synthetic mooring lines for depths to 3000 meters. In Proceedings of Offshore Technology Conference. Houston, TX, USA, 2002. Doi: https://doi.org/10.4043/14246-MS.

DIN 3390. Calculation of load capacity of cylindrical gears; introduction and general influence factors. Standard, Beuth, 1987. Doi: https://doi org/10. $31030 / 2069785$.

DIN ISO 281. Rolling bearings - dynamic load ratings and rating life. Standard, Beuth, 2010. Doi: https: //doi.org/10.31030/1718715.

DVNGL. Modeling and analysis in marine operation. 2017. DVNGL-RP-N103.

Falkenberg, E., Yang, L., and hjem, V. Spring-dashpot simulations of polyester ropes: Validation of the syrope model. Offshore Technology; Offshore Geotechnics, 2019. 1. Doi: https://doi.org/10.1115/ OMAE2019-95469.

Fernandes, A. and Rossi, R. Distorted polyester lines for model testing of offshore moored platforms. Ocean Engineering, 2005. 32:817-825. Doi: https: //doi.org/10.1016/j.oceaneng.2004.10.008. 
Flory, J., Banfield, S., and Petruska, D. Defining, measuring, and calculating the properties of fiber rope deepwater mooring lines. In Offshore Technology Conference Proceedings. Houston, TX, USA, 2004. Doi: https://doi.org/10.4043/16151-MS.

Flory, J., Banfield, S., Petruska, D., and Leech, C. Computer model to predict long-term performance of fiber rope mooring lines. In Offshore Technology Conference Proceedings. Houston, TX, USA, 2005. Doi: https://doi.org/10.4043/17592-MS.

Francois, M. and Davies, P. Fibre rope deep water mooring: a practical model for the analysis of polyester systems. In Proceedings of Rio Oil \& Gas Conference. Rio de Janeiro, Brazil, 2000.

Hasselmann, K., Barnett, T., Bouws, E., Carlson, H., Cartwight, D., Enke, K., Ewing, J., Gienapp, H., Hasselmann, D., Kruseman, P., Meerburg, A., Muller, P., Olbers, D., Richter, K., Sell, W., and Walden, H. Measurements of wind-wave growth and swell decay during the joint north sea wave project (jonswap). Ergnzungsheft zur Deutschen Hydrographischen Zeitschrift Reihe, 1973.

Huang, W., Li, B., and Chen, X. An investigation of dynamic stiffness changing with loading cycles on taut mooring analysis. In Proceedings of the 25th International Ocean and Polar Engineering Conference. Kona, Hawaii, USA, 2015.

Kurtz, S. The UHMWPE Handbook: The Clinical Performance of UHMWPE in Hip Replacements. The Journal of Bone and Joint Surgery, 2004. Doi: https://doi.org/10.1016/ B978-012429851-4/50006-1.

Leech, C., Banfield, S., Overington, M., and Lemoel, M. The prediction of cyclic load behavior and modulus modulation for polyester and other large synthetic fiber ropes. In Oceans 2003 Conference Proceedings. San Diego, CA, USA, 2003. Doi: https: //doi.org/10.1109/OCEANS.2003.178056.

Liu, H., Huang, W., Lian, Y., and Li, L. An experimental investigation on nonlinear behaviors of synthetic fiber ropes for deepwater moorings under cyclic loading. Applied Ocean Research, 2014. 45:22-32. Doi: https://doi.org/10.1016/j.apor.2013.12.003.
MacGregor. Offshore cranes. 2019. URL https: //www . macgregor.com/Products/products/ offshore-and-subsea-load-handling/ offshore-cranes/. Accessed: 2020-03-02.

Miner, M. Cumulative damage in fatigue. Journal of Applied Mechanics, 1945. 12:159-164.

Neumann, S., Wll, L., Jacobs, G., Feldermann, A., and Strassburger, F. Modular system modeling for quantitative reliability evaluation of technical systems. Modeling, Identification and Control: A Norwegian Research Bulletin, 2016. 37:19-29. Doi: https://doi.org/10.4173/mic.2016.1.2.

SNAME. Nomenclature for treating the motion of a submerged body through a fluid. Technical Report Bulletin 1-5. Society of Naval Architects and Marine Engineers, 1950.

Wibner, C., Versavel, T., and Masetti, I. Specifying and testing polyester mooring rope for the barracuda and caratinga fpso deepwater mooring systems. In Proceedings of Offshore Technology Conference. Houston, TX, USA, 2003. Doi: https: //doi.org/10.4043/15139-MS.

Wll, L., Jacobs, G., and Feldermann, A. Sensitivity analysis on the reliability of an offshore winch regarding selected gearbox parameters. Modeling, Identification and Control: A Norwegian Research Bulletin, 2017a. 38:51-58. Doi: https://doi.org/ 10.4173/mic. 2017.2.1.

Wll, L., Jacobs, G., and Kramer, A. Lifetime calculation of irregularly oscillating bearings in offshore winches. Modeling, Identification and Control: A Norwegian Research Bulletin, 2018. 39:61-72. Doi: https://doi.org/10.4173/mic.2018.2.2.

Wll, L., Schick, K., Jacobs, G., Kramer, A., and Neumann, S. Reliability evaluation of drivetrains: Challenges for offhighway machines. System Reliability, 2017b. Doi: https://doi.org/10.5772/ intechopen.70280. 